\title{
ESTUDO DA CINÉTICA DE CRESCIMENTO DOS CRISTAIS DE SORO DE LEITE PERMEADO ATRAVÉS DA CRISTALIZAÇÃO
}

\author{
M. C. R. FALCOMER ${ }^{1}$, G. A. TEIXEIRA ${ }^{1,2}$ e R. A. MALAGONI ${ }^{1}$ \\ ${ }^{1}$ Universidade Federal de Uberlândia, Faculdade de Engenharia Química \\ ${ }^{2}$ Universidade Federal do Triângulo Mineiro, Departamento de Engenharia de Alimentos \\ E-mail para contato:malagoni@feq.ufu.br
}

\begin{abstract}
RESUMO - A cristalização é uma operação unitária de extrema importância no ramo da Engenharia Química por ser considerada uma técnica vantajosa para a produção de sólidos puros a partir de soluções impuras. Sua principal vantagem é proporcionar características desejáveis ao produto final, como pureza e uniformidade. O soro de leite permeado é o produto obtido através da remoção parcial da proteína do soro de leite. É produzido através de filtração por membranas, seguido de concentração por evaporação e posterior desidratação. No sentido de estudar a cinética de crescimento dos cristais utilizando este soro, foram realizados ensaios isotérmicos $\left(50^{\circ} \mathrm{C}\right)$ em fase densa, utilizando-se sementes de lactose produzidas a $7^{\circ} \mathrm{C}$. As variáveis de processo (adimensional de vibração, tempo de operação e grau de supersaturação) foram determinadas através da otimização em um planejamento composto central. As análises de tamanho dos cristais serão realizadas por meio do equipamento Malvern MasterSizer.
\end{abstract}

\section{INTRODUÇÃO}

O processo de cristalização pode ser dividido em três etapas. A primeira fase do fenômeno de cristalização é a geração da força motriz, conhecida como supersaturação, obtida pela evaporação do solvente ou resfriamento do sistema. A saturação de uma solução é alcançada quando nela está presente a máxima quantidade de soluto que aquela quantidade de solvente pode dissolver. Sendo assim, a supersaturação da solução é obtida quando se acrescenta qualquer quantidade de soluto superior à quantidade de saturação, sem que ocorra a precipitação do soluto em questão. A segunda etapa do processo de cristalização é a nucleação. Esta pode ocorrer de forma ocasional, resultado da associação aleatória de moléculas de soluto em razão do movimento caótico da solução. Neste estágio, o aglomerado de moléculas de soluto recebe o nome de embrião. Este núcleo é primordial no processo de formação de cristais e, deve possuir um arranjo estável de moléculas de soluto em uma estrutura uniforme e ordenada, para dar origem a um cristal com forma regular. O crescimento dos cristais é a última etapa do processo de cristalização. O aumento de tamanho das partículas (cristal) está relacionado com duas etapas, a etapa difusional em que o soluto migra da solução para a interface de uma camada de adsorção, e a etapa seguinte em que as moléculas se acoplam ao retículo cristalino, numa reação de primeira ordem (Finzer e Martins, 2011).

Segundo mostraram Thomson e Dahleh (1998), o estudo da vibração está relacionado com os 


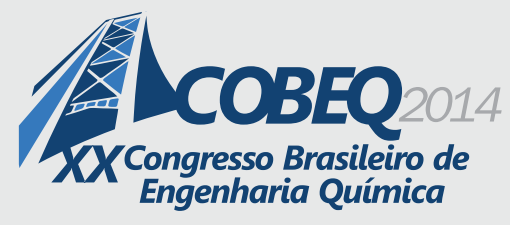

19 a 22 de outubro de 2014
Florianópolis/SC

movimentos oscilatórios de corpos e as forças associadas a eles e qualquer corpo que possua massa e elasticidade é capaz de vibrar. Na natureza existem dois tipos de vibração: natural, que ocorre quando um sistema oscila sob a ação de suas próprias forças, ou seja, sem a atuação de forças externas, e a forçada. O sistema sob vibração natural vibra em uma de suas frequências naturais, nas quais, são propriedades de um sistema dinâmico estabelecido pela sua distribuição de massa e tenacidade. Já a vibração forçada exige uma excitação provocada por forças externas que levam o sistema a vibrar na mesma frequência da excitação.

Para obtenção de cristais de dimensões apreciáveis, não se deve utilizar agitação de alta intensidade, mas tipos diferentes de agitação, de forma a facilitar a distribuição espacial dos cristais e a difusão do soluto para a superfície dos cristais (Finzer e Martins, 2011).

O permeado de soro é um subproduto da produção de proteína de leite concentrada a partir do soro de queijo. Os principais componentes do soro de leite permeado são água, lactose, aminoácidos e minerais. A elevada demanda química de oxigênio do permeado de soro faz com que o seu descarte represente um problema significativo de poluição. Devido a isso, sua eliminação torna-se dispendiosa para os fabricantes de queijo, apesar das várias técnicas disponíveis para isso (Nykänenet al., 1998). Segundo estudos de Cox e MacBean (1977), devidoao seu elevado teor de lactose, o permeado de soro pode ser usado como um meio de base da cultura para a produção de ácido láctico.

Segundo Brito (2007), a lactose é um produto de grande interesse industrial e não é produzida atualmente no Brasil, sendo sua importação de cerca de 5000 toneladas por ano em média nos últimos anos. Embora a lactose possa ser sintetizada, ela é obtida principalmente a partir de resíduos da produção de queijos e outros derivados do leite. Além disso, o reaproveitamento de resíduos de processos alimentícios é bastante viável, já que o grau de pureza em processos de cristalização é alto e o índice de contaminação é praticamente nulo. De acordo com Bem-Hassan e Ghaly (1994), a lactose é um importante componente para diversos processos biotecnológicos e também para as indústrias farmacêuticas e alimentícias.

Desta forma, o presente trabalho visa estudar o processo de cristalização da lactose a partir do permeado de soro em pó, bem como a cinética de crescimento dos cristais de lactose ao longo do processo.

\section{MATERIAL E MÉTODOS}

Para este trabalho, foram realizados três testes de cristalização. Todos foram realizados seguindo-se os mesmos procedimentos e mesmas condições de operação. Para o preparo da solução que foi colocada no cristalizador, utilizou-se permeado em pó da marca Sooro.

\subsection{Preparo da Solução}

Para cada experimento de cristalização, foi preparada uma solução de permeado em pó em água destilada. A proporção foi de $8,616 \times 10^{-2} \mathrm{~kg}$ de permeado em pó para $1,0 \times 10^{-1} \mathrm{~kg}$ de água. As pesagens foram feitas em uma balança analítica (Gehaka AG-200) devidamente calibrada. 
Após a solução ser homogeneizada, a mesma foi submetida a aquecimento em um banho termostatizado (TECNAL, TE - 184) até atingir a temperatura de $74^{\circ} \mathrm{C}$. Após a solubilização completa, $2,80 \times 10^{-1} \mathrm{~L}$ da solução final foi colocada no interior do cristalizador.

\subsection{Cristalização}

Primeiramente, o sistema que se encontrava a $74^{\circ} \mathrm{C}$ foi submetido a um resfriamento até atingir a temperatura de $50^{\circ} \mathrm{C}$. Tal temperatura foi justificada por Shi et al. (2006). Assim que a estabilidade térmica foi atingida, a vibração foi ajustada para $315 \mathrm{rpm}$. Então, foram adicionados $5,0 \times 10^{-2} \mathrm{~kg}$ de sementes de lactose monohidratada comercial (Granulac 200) da marca MEGGLE. Tais sementes haviam sido preparadas previamente. O tempo de operação dos testes foi de 2 horas e 11 minutos. As condições de operação foram testadas em um trabalho anterior.

Retirada de amostras: Durante o tempo de operação, foram retiradas amostras da solução diretamente do interior do cristalizador para análise. Para isto, utilizou-se uma pêra de sucção e uma pipeta volumétrica de $3 \mathrm{~mL}$. Após a retirada da amostra, esta foi submetida a um processo de filtração utilizando-se um funil com tela de separação 600 mesh conectado a uma bomba de vácuo (Quimis, 355 B2). O funil e a bomba de vácuo foram interligados por um kitassato de segurança. Os cristais foram lavados com duas borrifadas de álcool 95\%. Finalmente, os cristais foram colocados em uma estufa (Medicate, MD 1.3) a $60^{\circ} \mathrm{C}$ por um período de $12 \mathrm{~h}$ para que a umidade fosse removida. As amostras dos cristais foram pesadas e seu diâmetro analisado. A amostra líquida separada pelo funil teve o teor de lactose analisado posteriormente.

Cristalizador:O cristalizador utilizado foi construído em aço inoxidável. Ele possui formato tronco-cônico e é encamisado, além de ser vedado na parte inferior por uma membrana sintética flexível de borracha. No interior do cristalizador há um agitador com dois discos perfurados. Além do cristalizador, o aparato é composto por um motor de corrente alternada. Tal motor gera energia, que é transmitida ao leito por uma haste oscilatória e pelo excêntrico. Há ainda um banho termostatizado, que tem por função manter a temperatura no cristalizador. Para controlar a rotação do excêntrico, é utilizado um inversor de frequência e para medir a temperatura diretamente no interior do cristalizador, utiliza-se um termopar. O aparato completo está ilustrado pela Figura 1. 


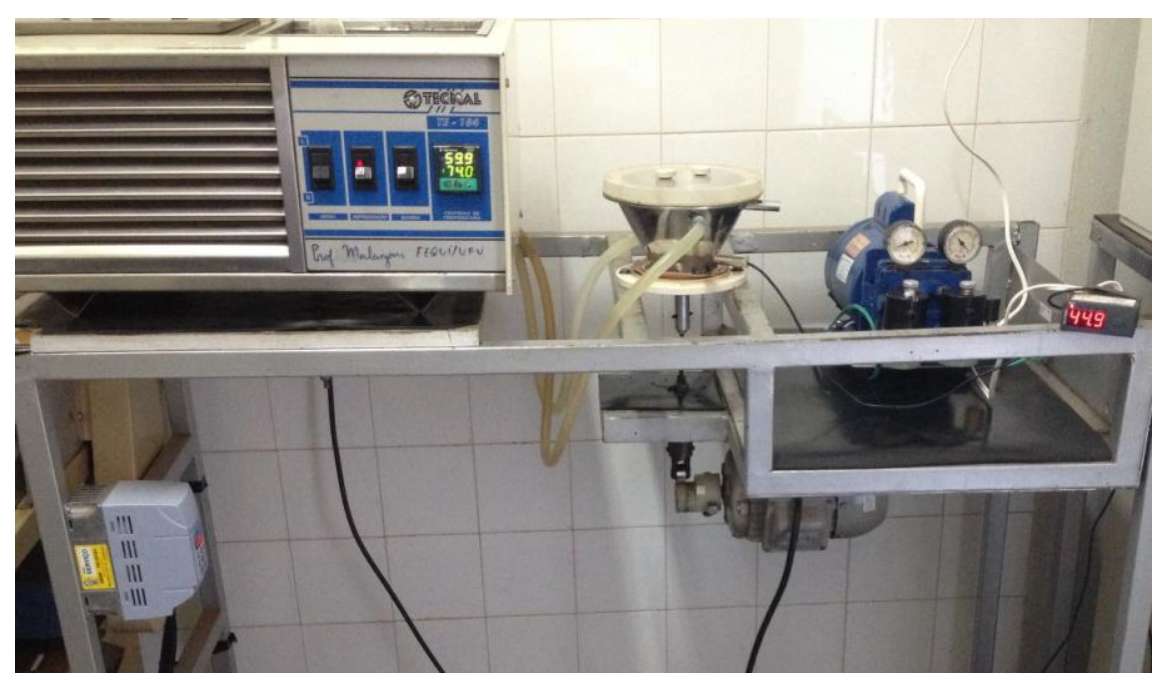

Figura 1 - Equipamento utilizado para os testes de cristalização.

\subsection{Análise do diâmetro de Sauter dos cristais}

Para avaliar o crescimento dos cristais ao longo do processo de cristalização, as amostras foram submetidas a uma análise via Malvern MasterSizer 2000. O fluido dispersante do sistema particulado utilizado foi o etanol.

\subsection{Análise doteor de lactose das amostras líquidas}

O teor de lactose das amostras líquidas foi medido segundo o Método do Ácido Dinitrossalicílico (DNS).

Preparo da solução de DNS: Para o preparo do reagente DNS adicionou-se $50 \mathrm{~mL}$ de hidróxido de sódio $(\mathrm{NaOH})$ com concentração $2 \mathrm{~mol} / \mathrm{L}$ a $2,5 \mathrm{~g}$ de ácido dinitrossalicílico e $125 \mathrm{~mL}$ de água destilada, agitando até a dissolução completa. Então, foram adicionados $75 \mathrm{~g}$ de sal de Rochelle (tartarato de sódio e potássio) e completou-se o volume da solução até 250 mL (Miller, 1959).

Método do Ácido Dinitrossalicílico: As amostras foram diluídas com água destilada em uma relação de 1:200. Após isto, 0,5 mL destas amostras foram colocadas em tubos Follin $\mathrm{Wu}$, juntamente com $1 \mathrm{~mL}$ da solução de DNS. Em seguida, as soluções foram tampadas e colocadas no banho termostatizado a $90^{\circ} \mathrm{C}$ por cinco minutos. Então, os tubos foram resfriados com água fria corrente, e o volume final de 12,5 $\mathrm{mL}$ foi completado com água destilada.

Análises espectrofotométricas: Após serem submetidas ao Método do DNS, as amostras foram analisadas no espectrofotômetro $\operatorname{com} \lambda=540 \mathrm{~nm}$. O branco utilizado foi uma amostra tratada segundo o mesmo método das demais, utilizando-se água destilada ao invés da amostra diluída. 


\section{RESULTADOS E DISCUSSÃO}

A massa das amostras cristalinas após 12 h de secagem na estufa se encontram dispostas na Tabela 1.A variável Tempo expressa em minutos nas tabelas representa o tempo em que cada amostra foi tirada do cristalizador. A amostra final foi retirada ao final de todo o processo.

Tabela 1 - Massa das amostras cristalinas

\begin{tabular}{|c|c|c|c|c|}
\hline Amostra & Teste 1 Massa $(\mathrm{g})$ & Teste 2 Massa $(\mathrm{g})$ & Teste 3 Massa $(\mathrm{g})$ & Tempo (min) \\
\hline 1 & 0,655 & 0,583 & 0,597 & 20 \\
\hline 2 & 0,728 & 0,619 & 0,633 & 50 \\
\hline 3 & 0,668 & 0,715 & 0,665 & 80 \\
\hline 4 & 0,794 & 0,774 & 0,705 & 110 \\
\hline 5 & 0,798 & 0,667 & 0,689 & 131 \\
\hline Final & 0,801 & 0,863 & 0,748 & - \\
\hline
\end{tabular}

Conforme era esperado, observa-se nos três testes a tendência de crescimento da massa das amostras ao longo do tempo. Isto é consequência do crescimento dos próprios cristais.

As análises feitas no Malvern Mastersizer 2000 forneceram o diâmetro de Sauter dos cristais. Tais resultados estão apresentados na Tabela 2, que apresenta o diâmetro médio dos três experimentos e o desvio padrão para cada tempo.

Tabela 2 - Diâmetro de Sautermédio dos cristais

\begin{tabular}{|c|c|c|}
\hline \multicolumn{1}{|l|}{ Amostra } & $\mathrm{D}_{\mathrm{sM}} \pm \alpha(\mu \mathrm{m})$ & Tempo $(\mathrm{min})$ \\
\hline 1 & $6,236 \pm 0,208$ & 20 \\
\hline 2 & $5,781 \pm 0,511$ & 50 \\
\hline 3 & $6,098 \pm 0,874$ & 80 \\
\hline 4 & $6,238 \pm 0,561$ & 110 \\
\hline 5 & $5,786 \pm 0,453$ & 131 \\
\hline Final & $5,718 \pm 0,507$ & - \\
\hline
\end{tabular}

Nota-se que não houve uma regularidade notável com relação ao tamanho dos cristais em nenhum dos três testes. Apesar disso, é possível perceber de uma forma geral que a tendência foi de decrescimento dos mesmos.

As análises de lactose foram realizadas segundo o método já descrito.As absorbâncias médias obtidas estão contidas na Tabela 3 e a concentração de lactose de cada amostra, na Tabela 4. A amostra inicial foi coletada assim que as sementes foram inseridas na solução. 
Tabela 3 - Absorbância média das amostras líquidas

\begin{tabular}{|c|c|c|}
\hline Amostra & $A_{\mathrm{m}} \pm \beta$ & Tempo (min) \\
\hline Início & $0,482 \pm 0,055$ & - \\
\hline 1 & $0,468 \pm 0,037$ & 20 \\
\hline 2 & $0,435 \pm 0,024$ & 50 \\
\hline 3 & $0,431 \pm 0,030$ & 80 \\
\hline 4 & $0,419 \pm 0,032$ & 110 \\
\hline 5 & $0,452 \pm 0,029$ & 131 \\
\hline
\end{tabular}

Tabela 4 - Concentração média de lactose das amostras líquidas

\begin{tabular}{|c|c|c|}
\hline Amostra & $C_{\mathrm{m}} \pm \gamma(\mathrm{g} / \mathrm{L})$ & Tempo (min) \\
\hline Início & $463,70 \pm 64,51$ & - \\
\hline 1 & $450,61 \pm 47,68$ & 20 \\
\hline 2 & $419,76 \pm 35,53$ & 50 \\
\hline 3 & $416,02 \pm 41,13$ & 80 \\
\hline 4 & $404,80 \pm 43,00$ & 110 \\
\hline 5 & $435,66 \pm 40,20$ & 131 \\
\hline
\end{tabular}

A Equação 1, obtida em um trabalho anterior através da construção de uma curva de calibração, descreve a relação entre a absorbância média e a concentração de lactose. É possível notar que as concentrações de lactose das amostras também não apresentaram uma regularidade muito precisa, mas, de uma forma geral, a tendência apresentada é de decrescimento. Isto se justifica pelo fato de a lactose que decresce na solução ser agregada ao cristal, cuja tendência é de crescimento.

$$
A_{m}=0,2158 C-0,0143
$$

\section{CONCLUSÃO}

A partir dos dados obtidos com este trabalho, foi possível notar que, apesar de a massa total das amostras cristalinas ter aumentado no geral ao longo tempo, o diâmetro de Sauter dos cristais diminuiu. Isto pode ser explicado por uma possível quebra dos cristais ao longo do experimento, causando a formação de novos núcleos. Além disto, foi possível estabelecer uma relação entre a concentração de lactose da amostra líquida e a massa da amostra cristalina. Quanto maior a concentração, mais lactose está presente na solução e, consequentemente, menos nos cristais, causando uma redução na massa da amostra. Isso foi verificado na maioria das amostras.

\section{NOMENCLATURA}

$A_{m} \quad$ Absorbância média 
$C_{m} \quad$ Concentração média de lactose $(\mathrm{g} / \mathrm{L})$

$D_{s M} \quad$ Diâmetro de Sauter médio $(\mu \mathrm{m})$

$\alpha \quad$ Desvio padrão do diâmetro de Sauter $(\mu \mathrm{m})$

$\beta \quad$ Desvio padrão da absorbância

$\gamma \quad$ Desvio padrão da concentração $(\mathrm{g} / \mathrm{L})$

$\lambda \quad$ Comprimento de onda (nm)

\section{REFERÊNCIAS}

BEM-HASSAN, R. M.; GHALY, A. E. Continuous propagation of kluyveromycesfagilis in cheese whey for pollution potential reduction.Applied Biochemistry and Biotechnology, v. 4/7, p. 89-105, 1994.

BRITO, A. B. N. Estudo da cristalização de lactose em diferentes solventes. Tese (Doutorado) Universidade Federal de São Carlos. São Carlos, 2007.

COX, G. C.; MACBEAN, R. D. Lactic acid production by Lactobacillus bulgaricus in supplemented whey ultrafiltrate. Aust J Dairy Technol, v. 32, p. 19-22, 1977.

FINZER, J. R. D.; MARTINS, J. R. Cristalização de lactose. FAZU emRevista, v. 8, 2011.

MILLER, G. K. Use of dinitrosalicylic acid reagent for determination of reducing sugar. Analytical Chemistry, v. 31, p. 426-428, 1959.

NYKÄNEN, A.; LAPVETELAINEN, A.; HIETNEN, R. M.; KALLIO, H. The effect of acetic acid, nisin-whey permeates sodium chloride and related combinations on aerobic plate count and the sensory characteristics of rainbow trout. LWT - Food Science and Technology, v.3, p 286-290, 1998.

\section{AGRADECIMENTOS}

Agradecemos ao CNPq pela bolsa de Doutorado, à SESu (Secretaria de Educação Superior) pela bolsa do PET, ao Prof. Dr. Carlos Henrique Ataíde por disponibilizar o Malvern MasterSizer para quantificar os cristais de lactose e à FEQUI/UFU pela estrutura fornecida para a realização da pesquisa no Laboratório de Cristalização. Agradecemos também à Fundação de Amparo à Pesquisa do Estado de Minas Gerais (FAPEMIG) pelos recursos concedidos no Projeto de Participação Coletiva em Eventos Técnicos-Científicos (PCE-00082-14). 